\title{
The ISS approach to the stability and robustness properties of nonautonomous systems with decomposable invariant sets: an overview
}

\author{
Paolo Forni ${ }^{\mathrm{a}}$, David Angeli ${ }^{\mathrm{b}}$ \\ ${ }^{a}$ Dept. of Electrical and Electronic Engineering, Imperial College London, UK \\ ${ }^{b}$ Dept. of Electrical and Electronic Engineering, Imperial College London, UK, and Dip. di Ingegneria dell'Informazione, University of \\ Florence, Italy
}

\begin{abstract}
This article is an overview of recent developments in the Input-to-State Stability framework, dealing in particular with the extension of the classical concept to systems with multiple invariant sets and possibly evolving on Riemannian manifolds. Lyapunov-based characterizations of the properties are discussed as well as applications to the study of cascaded nonlinear systems.
\end{abstract}

Keywords: Stability of nonlinear systems. Lyapunov methods.

\section{Introduction}

\subsection{Notion and importance of multistability}

The analysis of the stability and robustness properties of nonlinear open dynamical systems exhibiting a variety of non-trivial dynamical behaviors - multiple equilibria, periodicity, almost-periodicity, chaos - has great importance to several scientific disciplines ranging from mechanics, electronics, and physics to biology and neuroscience. Indeed, the phenomenon known as multistability, i.e. the coexistence of multiple attractors (with possibly very diverse nature) in a system of differential equations with a given set of parameters, is frequently found in many real physical systems. A thorough survey about the different domains in which multistability occurs has been presented in [40].

Among the reasons which make the study of multistable systems appealing from the perspective of system and control theory are a number of properties exhibited by such systems, as in the following. Firstly, they possess a "memory" of past states and, as components of larger systems, can act as switches or underlie relaxation oscillators [20]. Secondly, as essential components of many biological systems, they display functional flexibility in response to various transitory stimuli and, furthermore, they play a crucial role in cell differentiation and in the maintenance of phenotypic differences in the abscence of environmental distinctions [40]. Finally, again as components of larger networks, their dynamics might be at the very core of the mechanisms that entail information-processing or even intelligence [29]. The analysis of multistability thus appears as a key preliminary step towards what can be envisioned

Email addresses: p.forni14@imperial.ac.uk (Paolo Forni), d.angeli@imperial.ac.uk (David Angeli) as a new kind of control engineering, mainly oriented to applications in biology and neuroscience.

\subsection{Notion and importance of ISS}

In this context, stability notions for nonlinear systems with respect to exogeneous input disturbances become key tools. In fact, they allow to analyze stability of interconnected systems in terms of input-output gains of individual subsystems and, at the same time, they provide quantitative estimates of how each subsystem reacts to exogenous disturbances [9]. In this direction, the Input-to-State Stability (ISS) approach $[51,53,54]$ has extended the classical Lyapunov methods - traditionally used to establish internal stability properties - to systems with inputs and outputs, by exploiting energy-like functions in order to assess the stability and robustness of a system with respect to internal and external perturbations. We briefly recall here the classical notion of ISS together with a related notion called integral Input-to-State Stability (iISS) [10].

$(I S S)$. The definition of ISS implies the qualitative property of having the state eventually trapped in a ball whose radius is proportional to the magnitude of the input, and thus represents a measure of performance in the qualitative analog of "finite $\mathcal{L}^{\infty}$ to $\mathcal{L}^{\infty}$ induced norm". In particular, a general nonlinear system

$$
\dot{x}(t)=f(x(t), u(t)), y=h(x(t)),
$$

with state in $\mathbb{R}^{n}$, inputs in $\mathbb{R}^{m}$, and outputs in $\mathbb{R}^{p}$ is said to be input-to-state stable if it satisfies the following $\mathcal{L}^{\infty} \rightarrow$ $\mathcal{L}^{\infty}$ property: there exist some $\beta \in \mathcal{K} \mathcal{L}$ and $\gamma \in \mathcal{K}_{\infty}$ such that:

$$
|x(t)| \leq \beta(|x(0)|, t)+\gamma(\|u\|),
$$

for all inputs $u(\cdot)$ and all initial conditions $x(0) \in \mathbb{R}^{n}$. In (2), we have denoted the Euclidean norm with $|\cdot|$ and the $\mathcal{L}^{\infty}$ norm of signal $u(\cdot)$ with $\|u\|:=\sup _{t \geq 0}|u(t)|$.

April 15, 2016 
(iISS). As a weaker but still very meaningful notion of stability, the Integral Input-to-State Stability (iISS) has been introduced in [50]. The definition of iISS implies the qualitative property of small overshoot when disturbances have finite energy and thus represents a measure of performance in the qualitative analog of "finite $\mathcal{H}^{2}$ norm". System (1) is said to be $i I S S$ if there exist some $\beta \in \mathcal{K} \mathcal{L}$ and $\gamma \in \mathcal{K}_{\infty}$ such that:

$$
|x(t)| \leq \beta(|x(0)|, t)+\int_{0}^{t} \gamma(|u(s)|) d s,
$$

for all inputs $u(\cdot)$ and all initial conditions $x(0) \in \mathbb{R}^{n}$. The iISS property has been presented in terms of asymptotic time-domain characterizations, Lyapunov dissipation inequalities, and variants of the $\mathcal{L}^{2} \rightarrow \mathcal{L}^{\infty}$ estimate in references $[10,8,11]$.

Applications We are now going to mention a number of applications of the ISS property, and we refer the reader to [51] for an extensive presentation of the subject. Applications of the ISS property to the stabilization of classes of nonlinear systems [42], nonlinear cascades [49, 52] and feedback interconnections [33, 32]. Moreover, it has been shown in [49] that it is always possible for a GAS system to be refined - by means of feedback redesign - into a system which is ISS with respect to actuator disturbances. Applications of the iISS property are in the stabilization and disturbance attenuation of systems with bounded controls [34], nonlinear cascades [12], large-scale systems via decentralized output-feedback control [30], systems in block strict-feedback form via output regulation [28, 31], and hybrid switched systems [36].

\subsection{ISS and multistability: the "wrong" direction}

All classical formulations of the ISS property and its related properties [51] characterize stability properties in a global setting with respect to a single equilibrium at the origin and for systems defined in Euclidean space. In other words, classical ISS implies such system to evolve on the "flat" Euclidean space and such equilibrium to be globally asymptotically stable in absence of inputs. Interestingly enough, the classical definition of ISS allows in principle to formulate and characterize stability properties with respect to arbitrary compact invariant sets (and not simply equilibria), which automatically implies these arbitrary compact invariant sets to be both Lyapunov stable and globally attractive ${ }^{1}$. This requirement is applicable only to a class of compact invariant sets which consist of a single connected component [17]. Unfortunately, the invariant sets fail to consist of a single connected component in many behaviors of interest such as: almost global stability [44], bistability, multistability, periodic oscillations, convergence to almost-periodic attractors and to chaotic attractors. It is important to recall that stability analysis of each invariant solution can be conducted locally by means of standard tools. Nevertheless, as mentioned before, a number of applications and open problems in many domains of interest (theoretical biology, electro-mechanical systems, etc.) call for a global analysis of stability for these invariant solutions.

In regard to the global analysis of multistable systems, several approaches are available in the literature. In addition to the first monograph on the subject [25], the papers $[38,43]$ established the existence of Lyapunov functions for multistable systems evolving on a compact manifold. A modern approach is based on the aforementioned notion of almost global stability [44], that is convergence to an asymptotically stable equilibrium from all initial conditions except for those lying in a zero-measure set. The corresponding notion for nonautonomous systems is denoted as almost ISS [6]: the general nonlinear system (1) is said to be almost ISS with respect to an invariant compact set $\mathcal{A} \subset \mathbb{R}^{n}$ if $\mathcal{A}$ is locally asymptotically stable and there exists class- $\mathcal{K}_{\infty}$ function $\gamma$ such that:

$$
\forall u \forall \text { a.a. } \xi \in \mathbb{R}^{n} \limsup _{t \rightarrow+\infty}|x(t, \xi, u)|_{\mathcal{A}} \leq \gamma(\|u\|) .
$$

The key idea of this approach is to replace Lyapunov functions by suitable density functions and to impose a condition on the way these are propagated by the flow. The success of this approach has been validated both in terms of converse dual Lyapunov results fully characterizing the property [45], and in terms of software tools able to automatically find such density functions for certain classes of systems [41]. However, it has been shown in [5] that a fundamental limitation arises in the attempt of constructing density functions for a specific class of systems, such class encompassing the classical nonlinear pendulum model.

The difficulties in finding density functions pushed the research towards a complementary set of tools for the global analysis of stability and robustness in multistable systems [9]. The approach followed in [9] heavily relies on the stable and unstable manifold theory of dynamical systems and on their time-varying adaptation and, for this reason, provides a very fine structure to the stability properties of the invariant sets. In fact, the main result of the paper guarantees almost ISS for ultimately-bounded multistable systems whose linearization at each unstable equilibrium has at least one eigenvalue with positive real part. Even though a possible extension of this result to multiperiodic systems could be conjured by drawing the theory of Floquet multipliers in, such result would still be not only very hard to test analytically but also merely a sufficient result, thus not providing a full characterization of the almost ISS property.

Moving from the almost ISS framework, the paper [17] has shown that the most natural way of conducting a

\footnotetext{
${ }^{1}$ Non compact sets can somehow be tackled by considering output maps, and defining appropriate input-to-output stability properties. This is effective at least in the case of systems whose solutions are eventually trapped in a compact set (UBIBS systems) and for this case Lyapunov-like characterizations of the properties are possible $[48,55]$.
} 
global stability analysis for systems with multiple invariant sets is to relax the Lyapunov stability requirement rather than the global nature of the attractivity property. This intuition has led to a new line of research which starts from the characterizations of ISS for this class of systems in terms of usual Lyapunov-like inequalities [7], thus generalizing the classical ISS theory as well as the already-mentioned literature on autonomous flows evolving on compact manifolds [38]. This paper provides an overview of this current line of research and its related novel contributions. Furthermore, we would like to point out that the developed theory has already shown to be of great potential interest for applications in the domain of power systems $[19,18]$ and robotics [26].

\subsection{Notation}

The Riemannian distance between two points $x_{1}, x_{2}$ of a Riemannian manifold $M$ will be denoted with $\mathfrak{d}\left[x_{1}, x_{2}\right]$. We will denote the distance between signals $X_{1}, X_{2}: \mathbb{R}_{\geq 0} \rightarrow$ $M$ with $\mathfrak{d}_{[0,+\infty)}\left[X_{1}(\cdot), X_{1}(\cdot)\right]:=\sup _{t \geq 0} \mathfrak{d}\left[X_{1}(t), X_{2}(t)\right]$. Given a set $S \subset M$ and a point $p \in M$, the set-point distance will be denoted as $|p|_{S}=\inf _{x \in S} \mathfrak{d}[p, x]$. Given a function $V: M \rightarrow \mathbb{R}_{\geq 0}$ and a compact connected set $S \subset M$, the set $\bigcup_{x \in S} V(x)$ will be denoted for short as $V(S)$. Given a continuously differentiable function $V$ : $M \rightarrow \mathbb{R}_{>0}$, the covector field $D V(x): M \rightarrow T^{\star} M$ will denote the differential of $V$ evaluated at $x$, according to Definition 4.2.5 in [1]. Standard notation for comparison functions is as follows: a class- $\mathcal{K}$ function $\alpha: \mathbb{R}_{\geq 0} \rightarrow \mathbb{R}_{\geq 0}$ will denote a continuous and strictly increasing function with $\alpha(0)=0$; if, furthermore, $\alpha$ is unbounded, then $\alpha$ is said to be a class- $\mathcal{K}_{\infty}$ function; a class- $\mathcal{K} \mathcal{L}$ function will denote any function $\beta: \mathbb{R}_{\geq 0} \times \mathbb{R}_{\geq 0} \rightarrow \mathbb{R}_{\geq 0}$ such that $\beta(\cdot, s) \in \mathcal{K}_{\infty}$ for each $t \geq 0$ and $\beta(r, \cdot)$ is strictly decreasing with $\lim _{t \rightarrow+\infty} \beta(r, t)=0$ for each $r \geq 0$.

\section{ISS for systems on manifolds and with a decom- posable invariant sets}

\subsection{Recalls on dynamical systems on manifolds}

We start our analysis by recalling standard notions about dynamical systems evolving on manifolds $[16,13,1]$. Let $M$ be an $n$-dimensional connected and geodesically complete Riemannian manifold without boundary and let $\mathfrak{g}$ be the Riemannian metric associated with $M$. A manifold is said to be connected if it is not the union of two disjoint open sets, and is said to have no boundary if every point belonging to $M$ has a neighborhood which is homeomorphic to $\mathbb{R}^{n}$. The property of geodesical completeness is the property of every maximal geodesic $\gamma(t)$ on $M$ being extendible for all $t \in \mathbb{R}$. Geodesical completeness relates to the notion of completeness of $M$ as a metric space via the Hopf-Rinow theorem and implies compactness of all closed and bounded sets of $M$.

A vector field $X$ on $M$ is a section of the tangent bundle $T M$, that is a mapping $X: M \rightarrow T M$ such that $X(x) \in$
$T_{x} M$ for all $x \in M$. Typically, $X$ is required to satisfy local Lipschitz continuity $^{2}$. A curve $c$ at a point $m \in M$ is a $\mathcal{C}^{1}$ map from an open interval $I$ of $\mathbb{R}$ into $M$ such that $0 \in I$ and $c(0)=m$. We can then define an integral curve of $X$ at $m \in M$ as a curve $c$ at $m$ such that $\frac{d}{d t} c(t)=X(c(t))$ for each $t \in I$ (see [1, Chapter 4]). Then, a dynamical system on $M$ is precisely the law of motion of the form $\frac{d c}{d t}=X(c)$, $c(0)=m \in M$ whose integral curve is $c(t)$.

\subsection{The notion of decomposition and main assumption}

ISS is essentially an analytical theory which characterizes the input-to-state behavior of a system by means of Lyapunov-like dissipation inequalities and, for this reason, it can be seen as an energetic concept. Consistently with the dissipative behavior of the systems onto which classical ISS typically applies, we will focus our attention to the notion of decomposition of a compact invariant set and we will make clear that such decomposition must exhibit no cycles among its elements. In fact, a system cannot dissipate energy all along the trajectories connecting the atoms of a cycle. For this reason, whenever cycles are present and provided that ultimate boundedness of trajectories holds true, one might resort to coarser decompositions by embedding any homoclinic or heteroclinic cycle within a single atom of the decomposition. In particular, ultimate boundedness alone potentially allows one to resort to the coarsest decomposition, that is the one consisting of one unique atom, the Milnor attractor (see Remark 3), which is a globally asymptotically stable compact invariant set [7, Section III.C].

The notion of a decomposition with no cycles automatically rules out a number of conservative Hamiltonian systems which typically exhibit homoclinic/heteroclinic cycles to equilibria and also do not satisfy ultimate boundedness of trajectories. Another interesting example is provided by the Lorenz system, which can only be studied by embedding its chaotic attractor in a single invariant set, the Milnor attractor, comprising also the equilibria and all their connecting orbits.

Let $D$ be a closed subset of $\mathbb{R}^{m}$ containing the origin. Consider the system:

$$
\dot{x}(t)=f(x(t), d(t)),
$$

where $f(x, d): M \times D \rightarrow T_{x} M$ is a locally Lipschitz continuous mapping ${ }^{2}$, with state $x$ taking value in $M$ and $d(\cdot)$ any locally essentially bounded and measurable input signal taking values in $D$. We denote by $X(t, x ; d)$ the uniquely defined solution of (4) at time $t$ fulfilling $x(0)=x$ under the input $d(\cdot)$.

\footnotetext{
${ }^{2}$ A vector field $X$ on $M$ is called locally Lipschitz continuous if, for all compact sets $\mathcal{K} \subset M$, there exists a constant $C_{\mathcal{K}} \geq 0$ such that $\exp _{x}: T_{x} M \rightarrow M$ is bijective on $U$ and, for all $v \in U$, it holds $\left\|\mathcal{T}_{\exp _{x}(t v), 1,0} X\left(\exp _{x}(v)\right)-X(x)\right\|_{\mathfrak{g}_{x}} \leq C_{\mathcal{K}}\|v\|_{\mathfrak{g}_{x}}$. Here $\exp$ and $\mathcal{T}$ respectively denote the standard operators of exponential map and parallel transport on $M[16,13]$.
} 
The unperturbed system is defined by the following set of equations:

$$
\dot{x}(t)=f(x(t), 0) .
$$

Solutions of the unperturbed system (5) may fail to be defined for all $t \in \mathbb{R}$. However, we assume without loss of generality that any solution of (5) is globally defined backwards and forward in time ${ }^{3}$. At the same time, all $\alpha$ - and $\omega$ - limit sets of (5) are assumed to be compact (and possibly empty). Moreover, we assume that for any trajectory of (5) there exists $\omega$ - and $\alpha$ - limit $\operatorname{sets}^{4}$ which are compact (possibly empty).

Definition 2.1 ( $\mathcal{W}$-limit set). Let $\mathcal{W} \subset M$ be a compact invariant set containing all the $\alpha$-and $\omega$-limit sets of (5), that is $\alpha(x) \cup \omega(x) \subset \mathcal{W}, \forall x \in M$. Then the set $\mathcal{W}$ is called an $\mathcal{W}$-limit set for (5).

Note that an $\mathcal{W}$-limit set $\mathcal{W}$ is not required to satisfy any global attractivity property a priori.

Definition 2.2 (Decomposition). Let $\mathcal{W} \in M$ be a compact and invariant set for (5). A decomposition of $\mathcal{W}$ is a finite, disjoint family of compact invariant sets $\mathcal{W}_{1}, \ldots, \mathcal{W}_{K}$ (the atoms of the decomposition) such that:

$$
\mathcal{W}=\bigcup_{i=1}^{K} \mathcal{W}_{i}
$$

For an atom $\mathcal{W}_{w}$ of the invariant set $\mathcal{W}$, its attracting and repulsing subsets are defined as follows:

$$
\begin{aligned}
& \mathcal{A}(\mathcal{W})=\left\{x \in M:|X(t, x, 0)|_{\mathcal{W}_{w}} \rightarrow 0 \text { as } t \rightarrow+\infty\right\}, \\
& \mathcal{R}(\mathcal{W})=\left\{x \in M:|X(t, x, 0)|_{\mathcal{W}_{w}} \rightarrow 0 \text { as } t \rightarrow-\infty\right\} .
\end{aligned}
$$

Define a relation on $\mathcal{W}_{i}$ and $\mathcal{W}_{j}$ by $\mathcal{W}_{i} \prec \mathcal{W}_{j}$ if $\mathcal{A}\left(\mathcal{W}_{i}\right) \cap$ $\mathcal{R}\left(\mathcal{W}_{j}\right) \neq \emptyset$.

Definition 2.3 ( $r$-cycle, 1-cycle, filtration). Let $\mathcal{W}_{1}, \ldots, \mathcal{W}_{K}$ be a decomposition of $\mathcal{W}$, then:

1. An $r$-cycle $(r \geq 2)$ is an ordered $r$-tuple of distinct indexes $i_{1}, \ldots, i_{r}$ such that $\mathcal{W}_{i_{1}} \prec \cdots \prec \mathcal{W}_{i_{r}} \prec \mathcal{W}_{i_{1}}$.

2. A 1-cycle is an index $i$ such that $\left[\mathcal{R}\left(\mathcal{W}_{i}\right) \cap \mathcal{A}\left(\mathcal{W}_{i}\right)\right]-$ $\mathcal{W}_{i} \neq \emptyset$.

3. A filtration ordering is a numbering of the $\mathcal{W}_{i}$ so that $\mathcal{W}_{i} \prec \mathcal{W}_{j} \Rightarrow i<j$.

${ }^{3}$ Without loss of generality, system (5) can be made backward and forward complete by slowing down the dynamics with $\dot{x}=$ $\frac{1}{1+|f(x)|_{\mathfrak{g}}} f(x)$.

${ }^{4}$ The definition of $\alpha$ - and $\omega$-limit sets is the following:

$\alpha(x):=\left\{y \in M: \lim _{t_{k} \rightarrow-\infty} X\left(t_{k}, x\right)=y\right.$ for some diverging $\left.\left\{t_{k}\right\}_{k \in \mathbb{N}}\right\}$, $\omega(x):=\left\{y \in M: \lim _{t_{k} \rightarrow+\infty} X\left(t_{k}, x\right)=y\right.$ for some diverging $\left.\left\{t_{k}\right\}_{k \in \mathbb{N}}\right\}$.
Existence of a filtration ordering rules out existence of any $r$ - or 1-cycle among the atoms of the decomposition. Existence of an $r$-cycle for (5) with $r \geq 2$ implies existence of a heteroclinic cycle, and existence of a 1-cycle implies existence of a homoclinic orbit. The converse implication is not true: one can always select one of the atoms as the given heteroclinic cycle / homoclinic orbit itself so as to delineate a coarser decomposition with no cycles.

Our main assumption on $\mathcal{W}$, which will be used throughout the paper, is the following:

Assumption 1 (No cycle condition). The set $\mathcal{W}$ admits a finite decomposition without cycles, $\mathcal{W}=\bigcup_{i=1}^{K} \mathcal{W}_{i}$ for some non-empty disjoint compact sets $\mathcal{W}_{i}$, which form a filtration ordering of $\mathcal{W}$, as detailed in Definitions 2.2 and 2.3.

Example. Consider the following system of differential equations:

$$
\left(\begin{array}{l}
\dot{x}_{1} \\
\dot{x}_{2}
\end{array}\right)= \begin{cases}f_{1}\left(x_{1}, x_{2}\right) & \text { for } x_{1} \leq-1 \\
\frac{1}{2}\left[\left(1+x_{1}\right) f_{1}+\left(1-x_{1}\right) f_{2}\right] & \text { for }-1<x_{1} \leq 1 \\
f_{2}\left(x_{1}, x_{2}\right) & \text { for } x_{1}>1\end{cases}
$$

with $f_{1}\left(x_{1}, x_{2}\right):=\left(\begin{array}{c}x_{1}+2 \\ x_{2}-x_{2}^{3}\end{array}\right)$

$$
\text { and } f_{2}\left(x_{1}, x_{2}\right):=\left(\begin{array}{c}
-x_{2}+\left(x_{1}-2\right)\left(1-\left(x_{1}-2\right)^{2}-x_{2}^{2}\right) \\
x_{1}-2+x_{2}\left(1-\left(x_{1}-2\right)^{2}-x_{2}^{2}\right)
\end{array}\right) \text {. }
$$

The $\mathcal{W}$-limit set for system $(6)$ is:

$$
\mathcal{W}=\{(-2,0),(-2,-1),(-2,1),(2,0), \Gamma\},
$$

with $\Gamma=\left\{\left(x_{1}, x_{2}\right) \in \mathbb{R}^{2}:\left(x_{1}-2\right)^{2}+x_{2}^{2}=1\right\}$. By studying each vector field $f_{1}, f_{2}$ separately, it is easy to observe that the phase portrait of (6), as depicted in Figure 1, displays no homoclinic/heteroclinic cycles and for this reason satisfies Assumption 1. A filtration ordering for $\mathcal{W}$ is given by:

$$
\begin{aligned}
& \mathcal{W}_{1}=\Gamma, \mathcal{W}_{2}=\{(2,0)\}, \mathcal{W}_{3}=\{(-2,1)\} \\
& \mathcal{W}_{4}=\{(-2,1)\}, \mathcal{W}_{5}=\{(-2,0)\}
\end{aligned}
$$

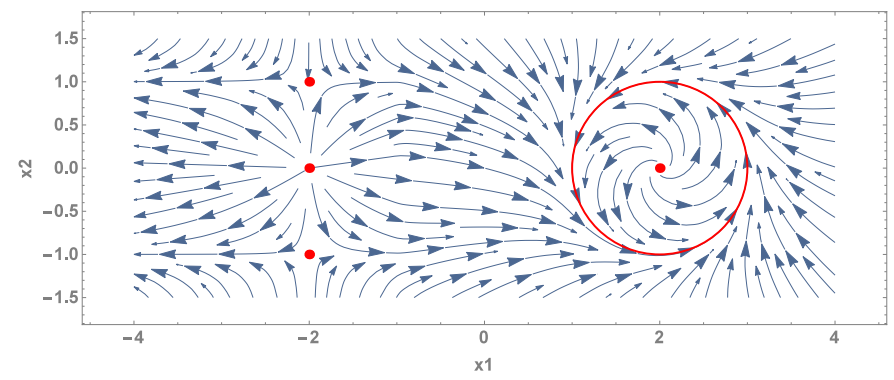

Figure 1: Phase plot of dynamics (6) in the coordinates $x_{1}, x_{2}$. The set $\mathcal{W}$ as in (7) is depicted in red. 


\subsection{Main result and discussion}

In the following, we will consider a particular robustness notion for system (4) denoted as asymptotic gain (AG) property. AG corresponds to the property of having the state of the system eventually trapped within a distance from the $\mathcal{W}$-limit set which is proportional to the infinity norm of the input. Similar notions include the practical AG (pAG) property, where the distance from the $\mathcal{W}$-limit set is proportional to the infinity norm of the input plus a constant, and the global zero-attractivity (0GATT) property that simply formalizes the property of $\mathcal{W}$ being attractive for all initial conditions in the state space whenever inputs are zeroed.

Definition 2.4 (pAG). The system (4) has the practical asymptotic gain (pAG) property if there exists $\eta \in \mathcal{K}_{\infty}$ and $q \geq 0$ such that for all $x \in M$ and all measurable essentially bounded inputs $d(\cdot)$ solutions are defined for all $t \geq 0$ and the following holds:

$$
\limsup _{t \rightarrow+\infty}|X(t, x ; d)|_{\mathcal{W}} \leq \eta(\|d\|)+q .
$$

If $q=0$, then we say that the asymptotic gain ( $\mathrm{AG}$ ) property holds. If (8) holds with $q=0$ and $\|d\|=0$, we say that the systems (4) and (5) satisfy the global zero-attractivity (0-GATT) property.

In the classical framework [51], ISS is equivalently characterized by the conjunction of the AG and 0-LS properties [54], with the latter property denoting Lyapunov stability of the origin whenever input are absent. The generalized notion of ISS for multistable systems in [7] replaces the 0LS requirement with Assumption 1 and is then formalized as

Definition 2.5. System (4) is said to be ISS with respect to the input $d$ and the invariant set $\mathcal{W}$ if and only if $\mathcal{W}$ satisfies Assumption 1 and (4) has the AG property.

Remark 1 One may object that Definition 2.5 formalizes a mere convergence property while ISS typically comprises Lyapunov stability and uniform attractivity. However, we have mentioned that the invariant set of a multistable system typically lacks Lyapunov stability and also uniform attractivity, and this is due to the so-called stickiness effect in proximity of anti-stable manifolds ${ }^{5}$. For this reason, the conjunction of the no-cycle assumption with the AG convergence property seems to be the most natural option for generalizing ISS to the domain of multistable systems. In particular, Definition 2.5 actually implies Lyapunov stability of $\mathcal{W}$ whenever $\mathcal{W}$ consists of a single connected component $\mathcal{W}_{0}$, and this result can be shown by proving that global attractivity of $\mathcal{W}_{0}$ plus absence of homoclinic trajectories to $\mathcal{W}_{0}$ are equivalent to global asymptotic stability of $\mathcal{W}_{0}$ [24]. Therefore, even if apparently no stability is entailed by the novel generalized definition of ISS, this very same definition is consistent with the standard one in (2) and actually naturally generalizes it.
We will then consider a characterization of the AG property in terms of a Lyapunov dissipation inequality. The reader is referred to [7] for an exhaustive presentation of this subject.

Definition 2.6 (ISS-Lyapunov function). $A \mathcal{C}^{1}$ function $V: M \rightarrow \mathbb{R}$ is a practical ISS-Lyapunov function for (4) if there exists $\mathcal{K}_{\infty}$ functions $\alpha_{1}, \alpha, \gamma$ and $q \geq 0$ such that, for all $x \in M$ and all $d \in D$, the following holds:

$$
\begin{aligned}
& \alpha_{1}\left(|x|_{\mathcal{W}}\right) \leq V(x) \\
& D V(x) f(x, d) \leq-\alpha\left(|x|_{\mathcal{W}}\right)+\gamma(|d|)+q
\end{aligned}
$$

If (10) holds with $q=0$, then $V$ is said to be an ISSLyapunov function. If, in addition, $V\left(\mathcal{W}_{i}\right)$ is a singleton for all $i \in\{1,2, \ldots, K\}$, then $V$ is said to be an ISSLyapunov function constant on invariant sets. If, furthermore, $D V(\mathcal{W})=0$, then $V$ is said to be an ISS-Lyapunov function flat on invariant sets.

Theorem 2.7 (Characterizations of ISS [7]). Consider a nonlinear system as in (4) and let $\mathcal{W}$ be an $\mathcal{W}$-limit set. System (4) is ISS with respect to input d and set $\mathcal{W}$ if and on if it admits a smooth ISS Lyapunov function constant on invariant sets. Furthermore, if system (4) satisfies Assumption 1, then the following facts are equivalent:

1. the system satisfies the $A G$ property;

2. the system satisfies the $p A G$ property;

3. the system admits a smooth ISS Lyapunov function flat on invariant sets;

4. the system admits an ISS Lyapunov function;

5. the system admits a practical ISS Lyapunov function.

Remark 2 There are two main differences between the Lyapunov characterization in Theorem 2.7 and the one in [53]. The first difference lies in the fact that the existence of a $\mathcal{K}_{\infty}$ function of $|x|_{\mathcal{W}}$ bounding $V$ from above is not necessary for ISS in the multistable context. In particular, the existence of such a bound would imply global asymptotic stability of $\mathcal{W}$, which in turn contradicts the absence of Lyapunov stability in systems having an invariant set with multiple disjoint components. In fact, the lack of such bound in (9) allows $V$ to take different values on the different components of the decomposition of $\mathcal{W}$. Since $V$ is strictly decreasing along the trajectories of the autonomous system (5) starting outside $\mathcal{W}, V$ takes high values on sources and low values on sinks. In other words, $V\left(\mathcal{W}_{i}\right)<V\left(\mathcal{W}_{j}\right)$ whenever $\mathcal{W}_{i} \prec \mathcal{W}_{j}$. The second difference is the absence in (8) of a $\mathcal{K} \mathcal{L}$ bound characterizing the convergence to the invariant set as in (2). Such bound cannot in fact exist in a system with an invariant set consisting of multiple disconnected components, and this is in fact due to the stickiness effect ${ }^{5}$.

5 Consider an invariant set $y \in M$ which is attractive for all initial conditions on its non-empty stable manifold $\mathcal{S}^{+}$. Fix $\varepsilon>0$ arbitrarily small. Define $T(x)$ as the capture time $T(x):=$ $\{T \geq 0: \mathfrak{d}[x, y] \leq \varepsilon, \forall t \geq T\}$. The stickiness effect is the phenomenon of having $T(\bar{x})$ growing arbitrarily large whenever $x$ approaches a given $\bar{x} \in M$, i.e. $\lim _{x \rightarrow \bar{x}} T(x)=+\infty$. 


\subsection{Examples}

\subsubsection{The single diode circuit}

Consider the example of a single diode electronic circuit described by the following set of differential equations [37]:

$$
\begin{aligned}
L \frac{d}{d t} i(t) & =E+d(t)-R i(t)-v(t) \\
C \frac{d}{d t} v(t) & =i(t)-f(v(t)),
\end{aligned}
$$

where $i, v, E, d, R, L, C$ respectively denote current flowing in the inductor, voltage across the capacitor, voltage supply, additive voltage supply, resistance, inductance, and capacitance, and where $i=f(v)$ denotes the nonlinear voltage/current characteristic of the negative resistor. Function $f(v)$ is smooth and satisfies the properties $f(0)=0$ and $f(v) v \geq 0$ for all $v \in \mathbb{R}$. Consider the function:

$V(i, v)=\frac{1}{2}\left(\frac{1}{L}-\frac{\lambda}{R}\right) \varphi_{i}(i, v)^{2}+\frac{1}{2 C} \varphi_{v}(i, v)^{2}+\frac{\lambda}{2 R}(E-v)^{2}+\lambda F(v)$,

with $\varphi_{i}(i, v):=E-R i-v, \varphi_{v}(i, v):=i-f(v), \lambda$ being a positive constant, and $F(v)=\int_{0}^{v} f(v)$. Observe that

$$
c_{0}\left(\varphi_{i}(i, v)^{2}+\varphi_{v}(i, v)^{2}\right) \leq V(i, v)
$$

for sufficiently small $c_{0}>0$. It has been shown in [37] that $V$ acts as a Lyapunov function for system (11) whenever $d(\cdot) \equiv 0$ and whenever $\lambda>0$ satisfies $-\frac{f^{\prime}(v)}{C}<\lambda<\frac{R}{L}$ for all $v \in \mathbb{R}$, with $f^{\prime}(v)=\partial f(v) / \partial v$. We are going to prove that $V$ is actually an ISS-Lyapunov function for (11). Indeed, by taking derivatives along solutions of (11), we obtain:

$$
\begin{aligned}
\dot{V}(i, v)= & -\frac{1}{L}\left(\frac{R}{L}-\lambda\right) \varphi_{i}(i, v)^{2}-\frac{1}{C}\left(\frac{f^{\prime}(v)}{C}+\lambda\right) \varphi_{v}(i, v)^{2}+ \\
& \frac{1}{L}\left(\lambda-\frac{R}{L}\right) \varphi_{i}(i, v) d+\frac{1}{L C} \varphi_{v}(i, v) d \\
\leq & -c_{1}\left(\varphi_{i}(i, v)^{2}+\varphi_{v}(i, v)^{2}\right)+c_{2} d^{2},
\end{aligned}
$$

for sufficiently small $c_{1}>0$, sufficiently large $c_{2}>0$, and $-\frac{f^{\prime}(v)}{C}<\lambda<\frac{R}{L}$. Define

$$
\mathcal{W}=\left\{(i, v) \in \mathbb{R}^{2}: \varphi_{i}(i, v)=\varphi_{v}(i, v)=0\right\} .
$$

Due to the special form of $\varphi_{i}$ and $\varphi_{v}, \mathcal{W}$ is compact, thus we can denote with $\mathcal{W}_{1}, \ldots, \mathcal{W}_{K}$ the $K$ non-empty disjoint connected compact components of $\mathcal{W}$. Observe that $\varphi_{i}(i, v)^{2}=\varphi_{v}(i, v)^{2}=0$ if and only if $(i, v) \in \mathcal{W}$ and that $\varphi_{i}(i, v)^{2} \rightarrow+\infty$ as $|(i, v)| \rightarrow+\infty$. Therefore, there exists two $\mathcal{K}_{\infty}$ functions $\alpha_{1}, \alpha$ such that inequalities (12) and (13) can be formulated as:

$$
\begin{array}{r}
\alpha_{1}\left(|(i, v)|_{\mathcal{W}}\right) \leq V(i, v) \\
\dot{V} \leq-\alpha\left(|(i, v)|_{\mathcal{W}}\right)+c_{2} d^{2} .
\end{array}
$$

By virtue of Theorem 2.7, we can conclude that the set $\mathcal{W}=\bigcup_{i=1}^{k} \mathcal{W}_{i}$ satisfies Assumption 1. Moreover, system (11) is ISS with respect to the set $\mathcal{W}$ and the disturbance $d$.
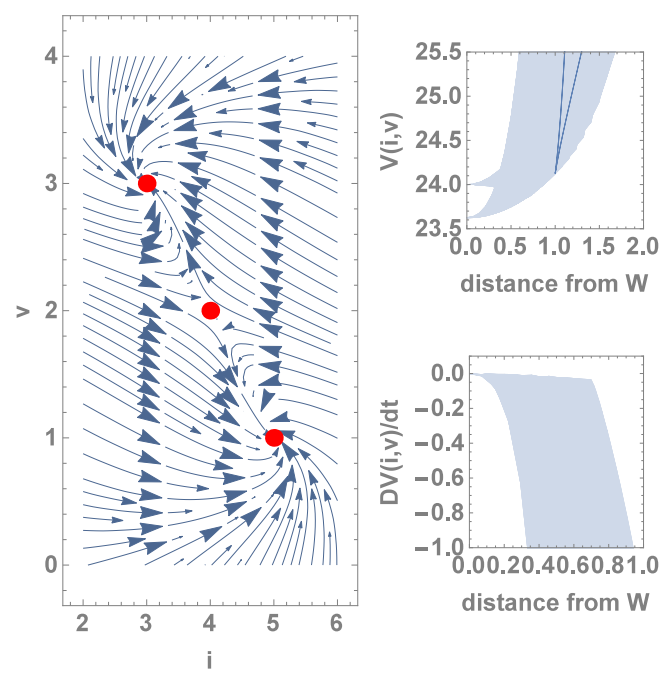

Figure 2: Phase portrait, $V$, and $\dot{V}$ of the single diode circuit with $E=6, R=1, C=2, L=1 / 2, \lambda=3 / 2, f(v)=10 v-6 v^{2}+v^{3}$. The corresponding invariant set is $\mathcal{W}=\{(3,3),(4,2),(5,1)\}$.

\subsubsection{Non decomposable invariant sets}

In the following example, we highlight the importance of Assumption 1. Consider the system:

$$
\dot{\theta}=1-\cos (\theta)+d
$$

with state $\theta$ identifying a point on the unit circle $\mathbb{S}=$ $\left\{(x, y) \in \mathbb{R}^{2}: x^{2}+y^{2}=1\right\}$. In the autonomous case $(d(t) \equiv$ $0)$, all solutions converge to the unique equilibrium $\theta=0$ (up to multiples of $2 \pi$ ). Nevertheless, the equilibrium $\theta=0$ is not asymptotically stable and, in fact, the system admits a homoclinic orbit to itself. This implies that the singleton $\{0\}$ does not satisfy Assumption 1 . This means that in order to apply Theorem 2.7, the $\mathcal{W}$-limit set has to be enlarged to the whole $\mathbb{S}$. However, one may wonder if the system can still be ISS with respect to the singleton $\{0\}$. To show that this is not possible we take a vanishing disturbance $d(t)=1 /(1+t)$ and we consider that:

$$
\begin{aligned}
\int_{0}^{+\infty}|\dot{\theta}(t)| d t & =\int_{0}^{+\infty}|1-\cos (\theta(t))+d(t)| d t \\
& \geq \int_{0}^{+\infty}|d(t)| d t=+\infty
\end{aligned}
$$

thus proving that solutions never stop describing full rotations around the circle. Hence, the choice of $\mathcal{W}=\mathbb{S}$ is in fact the tightest possible.

\section{Integral ISS}

\section{1. $\mathcal{L}_{2}$ notions of stability}

When characterizing the ISS property, a variety of equivalent notions are available in the literature. In particular, some results and applications have called for stability concepts which take into account "energy" information. In look for such integral variants of ISS, it has been shown in [50] that the estimate

$$
\int_{0}^{t} \alpha(|X(t, x ; d)|) d s=\xi(|x|)+\int_{0}^{t} \sigma(|X(t, x ; d)|) d s
$$


with $\alpha, \xi, \sigma \in \mathcal{K}_{\infty}$, not only shows finiteness of the $\mathcal{H}_{\infty}$ norm of system (4) but is also equivalent to ISS.

It turns out that similar estimates involving the energy of the input have actually been able to generate notions of stability in presence of inputs which are different both from stronger notions such as ISS and from weaker notions such as 0-GAS (global asymptotic stability with zero inputs). Generalizing the concept of $\mathcal{H}_{2}$ norm, that is the combination $\mathcal{L}_{2}$ to $\mathcal{L}_{\infty}$ as in 3 , yields in fact the integral ISS property [10]. Furthermore, it has been shown in [11] that mixed $\mathcal{L}_{p}$ to $\mathcal{L}_{q}$ norms with $p \neq q$, that is

$$
\left(\int_{0}^{t}(|X(t, x ; d)|)^{q} d s\right)^{\frac{1}{q}}=\left(|x|^{p}+\int_{0}^{t} \sigma(|X(t, x ; d)|)^{p} d s\right)^{\frac{1}{p}},
$$

are actually equivalent to integral ISS property.

\subsection{Main result and discussion}

In the following, we will show how the integral ISS concept naturally extends for systems with decomposable invariant sets. Once again, the characterization will be given in terms of asymptotic properties and Lyapunov dissipation inequalities. The reader is referred to [22] for an exhaustive presentation of this subject.

We start our characterization of integral ISS by introducing the UBEBS property in Definition 3.1 which portrays the behavior of having the distance of the state from the $\mathcal{W}$-limit set at all times growing no larger than the injected energy.

Definition 3.1 (UBEBS). System (4) is said to have the uniform bounded-energy bounded-state (UBEBS) property if, for some $\alpha, \gamma, \sigma \in \mathcal{K}_{\infty}$ and some positive constant $c_{\text {ubebs }}$, the following estimate holds for all $t \geq 0$, all $x \in M$ and all $d(\cdot) \in \mathcal{M}_{D}$ :

$$
\alpha(|X(t, x ; d)| \mathcal{W}) \leq \gamma\left(|x|_{\mathcal{W}}\right)+\int_{0}^{t} \sigma(|d(s)|) d s+c_{u b e b s}
$$

In the classical framework, integral ISS is actually equivalent to the conjunction of the UBEBS and 0-GAS properties [11]. Our generalized notion of ISS in [22] replaces the latter property with Assumption 1 and the 0-GATT property, and is formalized as follows.

Definition 3.2. System (4) is said to be integral ISS with respect to the input $d$ and the invariant set $\mathcal{W}$ if and only if $\mathcal{W}$ satisfies Assumption 1, and (4) has the 0-GATT and $U B E B S$ properties.

The very same reasons provided in Remark 1 allows the adoption of Definition 3.2 as a natural generalization of the standard notion of integral iISS in [50, 10].

It has been shown [11] that integral ISS entails convergence of the state whenever the energy of the input is finite under a given weighting gain. This behavior has proven to be especially useful in cascade systems [21] and continues to hold in the multistable settings, as follows.
Definition 3.3 (BESCS). System (4) is said to satisfy the Bounded Energy Strongly Converging State (BESCS) property if there exists a function $\tilde{\sigma} \in \mathcal{K}_{\infty}$ such that the following holds for all $x \in M$, and all $d(\cdot) \in \mathcal{M}_{D}$ :

$$
\int_{0}^{+\infty} \tilde{\sigma}(|d(s)|) d s<+\infty \Rightarrow \lim _{t \rightarrow+\infty}|X(t, x ; d)|_{\mathcal{W}}=0 .
$$

The following definitions represent several Lyapunovlike dissipation inequalities which equivalently characterize integral ISS in the multistable settings, as formally stated in Theorem 3.7.

Definition 3.4 (iISS-Lyapunov function). $A \mathcal{C}^{1}$ function $V: M \rightarrow \mathbb{R}$ is called an iISS-Lyapunov function for system (4) if there exist functions $\alpha_{1}, \alpha_{2}, \gamma \in \mathcal{K}_{\infty}$, and $c \geq 0$, and a continuous positive definite function $\alpha_{3}$ such that, for all $x \in M$,

$$
\alpha_{1}\left(|x|_{\mathcal{W}}\right) \leq V(x)
$$

and the following dissipation inequality hold for all $(x, d) \in$ $M \times D$ :

$$
D V(x) f(x, d) \leq-\alpha_{3}\left(|x|_{\mathcal{W}}\right)+\gamma(|d|) .
$$

If, in addition, $V\left(\mathcal{W}_{i}\right)$ is a singleton for all $i \in\{1,2, \ldots, K\}$, then $V$ is said to be an iISS-Lyapunov function constant on invariant sets. If, furthermore, $D V(\mathcal{W})=0$, then $V$ is said to be an iISS-Lyapunov function flat on invariant sets.

Definition 3.5 (Smooth dissipativity). System (4) is said to be smoothly dissipative if there exists a $\mathcal{C}^{1}$ function $V: M \rightarrow \mathbb{R}$, functions $\alpha_{1}, \alpha_{2}, \sigma \in \mathcal{K}_{\infty}$, a continuous positive-definite function $\alpha_{4}$, and a continuous output map $h: M \mapsto \mathbb{R}^{q}$, such that (17) holds and the following dissipation inequality holds for all $(x, d) \in M \times D$ :

$$
D V(x) f(x, d) \leq-\alpha_{4}(|h(x)|)+\sigma(|d|) .
$$

The following detectability notion will be useful in our main result about integral ISS.

Definition 3.6 (Weak zero-detectability). Given a continuous output map $h(x)$, system (4) is said to be weakly zero-detectable if $h(X(t, x ; 0)) \equiv 0$ for all $t \geq 0$ implies $|X(t, x ; 0)|_{\mathcal{W}} \rightarrow 0$ as $t \rightarrow+\infty$.

Theorem 3.7 (Characterizations of integral ISS [22]). Consider a nonlinear system as in (4) and let $\mathcal{W}$ be a $\mathcal{W}$ limit set satisfying Assumption 1. System (4) is integral ISS with respect to input d and set $\mathcal{W}$ if and only if it admits a smooth iISS-Lyapunov function constant on invariant sets. Furthermore, if system (4) satisfies Assumption 1, then the following facts are equivalent:

1. the system satisfies the zero-GATT and UBEBS properties;

2. existence of a smooth iISS-Lyapunov function flat on invariant sets; 
3. existence of an iISS-Lyapunov function;

4. existence of an output function that makes the system smoothly dissipative and weakly zero-detectable;

5. BESCS;

Remark 3 The proof of the necessity of existence of an iISS-Lyapunov function $V$ involves the understanding of two fundamental insights about zero-GATT and UBEBS properties. First insight is that zero-GATT implies the existence of a smooth function $U: M \rightarrow \mathbb{R}$, a $\mathcal{K}$ not $\mathcal{K}_{\infty}$ - function $\nu_{1}$, a $\mathcal{K}_{\infty}$ function $\delta$, and a positive definite function $\varpi$ such that:

$$
\begin{aligned}
& \nu_{1}\left(|x|_{\mathcal{W}}\right) \leq U(x) \\
& D U(x) f(x, d) \leq-\varpi\left(|x|_{\mathcal{W}}\right)+\delta(|d|),
\end{aligned}
$$

for all $x \in M$ and $d \in D$ (Section $\mathrm{C}$ in [7] and Lemma 3 in [22]). Since $\varpi$ might not be a proper function, dissipation at "larger" values of the state might not be able to properly counteract the injected energy. For this reason, zero-GATT alone does not guarantee boundedness of trajectories for bounded input signals. Second insight is that zero-GATT always guarantees the existence of a 0-GAS invariant set called the Milnor attractor (Section $\mathrm{C}$ in [7]) and defined as

$$
\mathcal{D}=\bigcup_{n=1}^{+\infty} \bigcap_{t \geq 0} X\left(t, M_{n}\right),
$$

where we have denoted with $M_{1} \subset M_{2} \subset \cdots \subset M$ a monotonically increasing sequence of compact subsets such that $M=\bigcup_{n=1}^{+\infty}$ int $\left\{M_{n}\right\}$. It turns out that 0-GAS of $\mathcal{D}$ together with UBEBS with respect to $\mathcal{W}$ implies the existence of an integral ISS Lyapunov function $W$ in the sense of classical integral ISS, that is

$$
\begin{aligned}
& \bar{\alpha}_{1}\left(|x|_{\mathcal{D}}\right) \leq W(x) \leq \bar{\alpha}_{2}\left(|x|_{\mathcal{D}}\right) \\
& D W(x) f(x, d) \leq-\bar{\alpha}_{3}\left(|x|_{\mathcal{D}}\right)+\bar{\sigma}(|d|)
\end{aligned}
$$

with $\bar{\alpha}_{1}, \bar{\alpha}_{2}, \bar{\sigma}_{1}$ being a $\mathcal{K}_{\infty}$ functions and $\bar{\alpha}_{3}$ being a positive definite function. Intuitively, we may say that the behavior of the system outside $\mathcal{D}$ is largely determined by dissipation (21) while the behavior inside $\mathcal{D}$ is determined by dissipation (20). Not unexpectedly, the integral ISS Lyapunov function of Definition 3.4 will be given by the sum of $U$ and $W$.

We bring to the attention of the reader that Remark 2 continues to hold true for Theorem 3.7 as well.

\subsection{Strong iISS}

ISS entails the so called bounded-input bounded-state (BIBS) property which typically makes ISS a very strong requirement in many applications of interest [14]. As a weaker requirement, but still matching several systems of practical interest, the notion of Strong iISS was introduced in [14] as an intermediate property, halfway between ISS and iISS. A strong iISS system is in fact an iISS system which exhibits the ISS behavior whenever the magnitude of the input is less or equal to a certain threshold. In the following, we will be focusing on a sufficient condition for Strong iISS in the context of multistable systems [22].

Definition 3.8 (Strong iISS). System (4) is said to be Strongly iISS if it has the properties:

- zero-GATT and UBEBS;

- asymptotic gain (AG) with respect to small inputs, namely there is a function $\eta \in \mathcal{K}$ and a positive constant $R$ such that:

$$
\|d\| \leq R \Rightarrow \limsup _{t \rightarrow+\infty}|X(t, x ; d)|_{\mathcal{W}} \leq \eta(\|d\|)
$$

for all $x \in M$ and all $d(\cdot) \in \mathcal{M}_{D}$.

Theorem 3.9 (Sufficient Lyapunov conditions for Strong iISS). Assume that there exists a proper $\mathcal{C}^{1}$ function $V$ : $M \rightarrow \mathbb{R}$, functions $\alpha_{1}, \alpha_{2} \in \mathcal{K}_{\infty}, \alpha \in \mathcal{K}$, a continuous positive-definite function $\gamma$, and a positive constant $c$ such that (17) holds together with the following dissipation inequality:

$$
D V(x) f(x, d) \leq-\alpha\left(|x|_{\mathcal{W}}\right)+\gamma(|d|)
$$

for all $(x, d) \in M \times D$. Then the system (4) is Strongly iISS.

\subsection{Examples}

\subsubsection{Tracking velocity fields in robotic manipulators}

Consider the general equations of motion for a robotic manipulator:

$$
M(q) \ddot{q}+C(q, \dot{q}) \dot{q}+G(q)=\tau+d
$$

with $q, \dot{q}, \ddot{q}$ the joint angles, velocities and accelerations, $M(q)$ the inertia matrix, $C(q, \dot{q})$ the Coriolis matrix, $G(q)$ the potential vector field, $\tau$ the vector of all available control torques, and $d$ disturbances occurring in the joint dynamics. Suppose that it is desired for the robotic manipulator to track a reference velocity field with desired reference dynamics:

$$
\dot{q}=f_{d}(q) .
$$

Let $\mathcal{W}_{q}$ denote the set of all invariant solutions of (25). Let $\mathcal{W}$ denote the following set:

$$
\mathcal{W}=\left\{\left(\begin{array}{c}
q \\
\dot{q}
\end{array}\right) \in \mathbb{S}^{n} \times \mathbb{R}^{n} \mid q \in \mathcal{W}_{q}, \dot{q}=f_{d}(q)\right\} .
$$

Suppose that for the desired reference dynamics (25) an iISS-Lyapunov function $\mu\left(q_{d}\right)$ exists, namely there exists $\mathcal{K}_{\infty}$ functions $\mu_{1}, \mu_{2}$, a continuous positive-definite function $\mu_{3}$ and a positive constant $c_{q}$ such that the following holds:

$$
\begin{array}{r}
\mu_{1}\left(\left|q_{d}\right| \mathcal{W}_{q}\right) \leq \mu\left(q_{d}\right) \leq \mu_{2}\left(\left|q_{d}\right| \mathcal{W}_{q}+c_{q}\right) \\
D \mu\left(q_{d}\right) f_{d}\left(q_{d}\right) \leq-\mu_{3}\left(\left|q_{d}\right| \mathcal{W}_{q}\right) .
\end{array}
$$


Let $F(q, \dot{q}):=\dot{q}-f_{d}(q)$. Consider the control law:

$$
\begin{aligned}
\tau= & -K F(q, \dot{q})-D \mu(q) \\
& +M(q)\left(D f_{d}(q) \dot{q}\right)+C(q, \dot{q}) f_{d}(q)+G(q),
\end{aligned}
$$

with a positive-definite gain matrix $K$. We want to prove that the closed-loop system obtained by applying the control law (27) to the plant (24), namely

$$
M(q) \dot{F}(q, \dot{q}, \ddot{q})+C(q, \dot{q}) F(q, \dot{q})+K F(q, \dot{q})+D \mu(q)=d,
$$

has the UBEBS and zero-GATT properties with respect to the invariant set $\mathcal{W}$ and the disturbance $d$. To this end, let $V(q, \dot{q})$ be the following Lyapunov function:

$$
V(q, \dot{q})=\mu(q)+\frac{1}{2} F(q, \dot{q})^{\top} M(q) F(q, \dot{q}) .
$$

Let the state $x$ be defined as $x:=\left(q^{\top}, \dot{q}^{\top}\right)^{\top}$. The distance from the invariant set $\mathcal{W}$ is then given by:

$$
|x|_{\mathcal{W}}:=\sqrt{|q|_{\mathcal{W}_{q}}^{2}+|F(q, \dot{q})|^{2}} .
$$

Due to $M(q)$ being the inertia matrix of a mechanical system, we can conclude that there exists functions $\alpha_{1}, \alpha_{2} \in$ $\mathcal{K}_{\infty}$ and a positive constant $c$ such that:

$$
\begin{aligned}
& \alpha_{1}\left(|x|_{\mathcal{W}}\right) \leq \mu_{1}\left(\left|q_{d}\right| \mathcal{W}_{q}\right)+\underline{\varepsilon}|F(q, \dot{q})|^{2} \leq V(q, \dot{q}) \\
& \leq \mu_{2}\left(\left|q_{d}\right| \mathcal{W}_{q}+c_{q}\right)+\bar{\varepsilon}|F(q, \dot{q})|^{2} \leq \alpha_{2}\left(|x|_{\mathcal{W}}+c\right) .
\end{aligned}
$$

By making use of skew-symmetry of $\dot{M}(q(t))-2 C(q(t), \dot{q}(t))$ in taking the derivative of $V$ along the solutions of (28), we obtain:

$$
\begin{aligned}
\dot{V}(q, \dot{q}) & =D \mu(q) \dot{q}-F(q, \dot{q})^{\top} K F(q, \dot{q})+F(q, \dot{q})^{\top} d \\
& \leq-\mu_{3}\left(|q|_{\mathcal{W}_{q}}\right)-c_{1}|F|^{2}+c_{2}|d|^{2}
\end{aligned}
$$

for sufficiently small positive constant $c_{1}$ and sufficiently large positive constant $c_{2}$. By setting $\alpha_{3}\left(|x|_{\mathcal{W}}\right)=\mu_{3}\left(|q|_{\mathcal{W}_{q}}\right)+$ $c_{1}|F|^{2}$, we can conclude that $V(q, \dot{q})$ is an iISS-Lyapunov function for system (28), therefore system (28) has the UBEBS and zero-GATT properties.

Remark 4 Note that, in case of $\mu_{3} \in \mathcal{K}_{\infty}$ (respectively $\mu_{3} \in \mathcal{K} \backslash \mathcal{K}_{\infty}$ ), system (28) would be ISS (respectively Strongly iISS, as in Theorem 3.9).

\subsubsection{The nonlinear pendulum}

Inequality (31) highlights the role of friction (respectively damping injection) in characterizing the stability properties of a general mechanical system in open-loop (in closed-loop). Consider the case of the nonlinear pendulum:

$$
\begin{aligned}
\dot{\theta} & =\omega \\
\dot{\omega} & =-a \sin (\theta)-b(\omega)+d,
\end{aligned}
$$

with state $x:=(\theta, \omega) \in \mathbb{S} \times \mathbb{R}$, disturbance $d$, positive constant $a$, and $b(\omega)$ denoting (possibly nonlinear) friction. In case of linear friction, i.e. $b(\omega)=b \omega$, the nonlinear pendulum has been proved to be ISS with respect to the input $d$ and the invariant set $\mathcal{W}=\{(0,0),(\pi, 0)\}$ in $[9$, $19,18]$. In case of vanishing friction, i.e. $b(\omega)=b \omega /(1+$ $\left.\omega^{2}\right)$, the nonlinear pendulum is iISS and not Strong iISS (Section V A in [22]). In case of friction saturation, i.e. $b(\omega)=b \omega^{2} /\left(1+\omega^{2}\right)$, the nonlinear pendulum is Strong iISS and the result is proved by using the same Lyapunov function as in $[9,19,18])$.

\section{Cascades}

\subsection{Introduction}

One of the most successful features of the classical ISS framework is its straightforward applicability to cascades of ISS systems [52]. The ease with which such result can be established is a direct consequence of two properties of ISS: the bounded-input bounded-state (BIBS) and the converging-input converging-state (CICS) properties.

In the next Subsections, we derive sufficient conditions for the ISS stability of the cascade system (33), when assuming the multistable behavior of the driving system (34a). Special attention will be paid to the modalities with which the filtration ordering of the cascade is inherited from the filtration orderings of each subsystem. The reader is referred to [23] for an exhaustive presentation of the subject.

\subsection{Multistability}

Let $M_{x}$ and $M_{z}$ be two connected Riemannian manifolds without boundary and having dimension $n_{x}$ and $n_{z}$ respectively. Let $D$ be a closed subset of $\mathbb{R}^{m}$ containing the origin. The subject of our study is the cascade system:

$$
\begin{aligned}
\dot{x}(t) & =g(x(t), d(t)) \\
\dot{z}(t) & =f(z(t), x(t)),
\end{aligned}
$$

where $g(x, d): M_{x} \times D \rightarrow T_{x} M_{x}$ and $f(z, x): M_{z} \times$ $M_{x} \rightarrow T_{z} M_{z}$ are two Lipschitz continuous mappings, and $d(\cdot)$ is any locally essentially bounded and measurable input signal taking values in $D$. We denote by $X(t, x ; d)$ the uniquely defined solution of (33a) at time $t$ fulfilling $X(0, x ; d)=x$, under the input $d(\cdot)$. In a similar way, we denote by $Z(t, z ; X)$ the uniquely defined solution of (33b) at time $t$ fulfilling $Z(0, z ; X)=z$, under the input $X(\cdot)$. Finally, we denote by $y=(x, z) \in M_{x} \times M_{z}$ the joint state and by $Y(t, y ; d)$ the uniquely defined solution of (33) at time $t$ fulfilling $Y(0, y ; d)=y$ under the input $d(\cdot)$. We also consider the unperturbed cascade system:

$$
\begin{aligned}
& \dot{x}(t)=g(x(t), 0) \\
& \dot{z}(t)=f(z(t), x(t)) .
\end{aligned}
$$

Let $\mathcal{W}_{x}$ denote a $\mathcal{W}$-limit set of the driving system (34a). The set $\mathcal{W}_{x}$ is assumed to satisfy the following

Assumption 2. (Multistability without cycles of driving system) The driving system (34a) satisfies the no-cycle condition and, moreover, each atom of the decomposition of $\mathcal{W}_{x}$ is a singleton, namely $\mathcal{W}_{x, i}=\left\{x_{i}\right\}$ with $x_{i} \in M_{x}$ for all $i=1, \ldots, K$. 
Remark 5 The assumption of having fixed points only in the decomposition of $\mathcal{W}_{x}$ can actually be removed, see [23, Section 5]. For ease of presentation, here we consider fixed points only.

We are looking for a $\mathcal{W}$-limit set which satisfies the no-cycle condition under the flow of the cascade system (34). In order to characterize such set in terms of its finest decomposition, we observe that, if ISS holds for subsystem (33a), all trajectories $X(t, x ; 0)$ eventually approach one of the $\omega$-limit sets in $\mathcal{W}_{x}$, namely one of the $x_{i}$ s. Therefore, it makes sense to study the stability of each "limit equation":

$$
\dot{z}(t)=F_{i}(z(t)):=f\left(z(t), x_{i}\right), \quad i=1, \ldots, K .
$$

Let $\mathcal{W}_{z}^{(i)}$ denote a $\mathcal{W}$-limit set of the limit equation $(35)$ for $i=1, \ldots, K$.

Assumption 3. (No-cycle condition of each limit equations) For all $i=1, \ldots, K$, each limit equation (35), thus each set $\mathcal{W}_{z}^{(i)}$ is decomposed in $K_{i}$ compact invariant subsets and satisfies the no-cycle condition.

A natural question that arises from this setting is whether the sought $\mathcal{W}$-limit set for (34) can be selected as the set:

$$
\mathcal{W}_{\Theta}:=\bigcup_{i=1}^{K}\left(\left\{x_{i}\right\} \times \mathcal{W}_{z}^{(i)}\right) \text {. }
$$

Indeed, we have the following result:

Theorem 4.1 (Cascades of ISS systems [23]). Let Assumptions 2 and 3 hold. If (H1) the driving system (33a) is ISS wrt input $d$ and set $\mathcal{W}_{x}$; (H2) the driven system (33b) is ISS wrt input $|x|^{1}$ and one of the sets $\mathcal{W}_{z}^{(i)}$; then (T1) $\mathcal{W}_{\Theta}$ qualifies as a $\mathcal{W}$-limit set for system (34) and admits a finite decomposition without cycles under the flow of (34); (T2) the cascade system (33) is ISS wrt inputd and the set $\mathcal{W}_{\Theta}$.

The same kind of result holds in case of Strongly iISS subsystems in the classical Euclidean setting [15]. To estabilish the same result in the multistable context, a preliminary observation is that compactness of the set $\mathcal{W}_{x}$ entails the existence of a function $\nu_{3} \in \mathcal{K}_{\infty}$ and a constant $c_{3}>0$ such that:

$$
|x| \leq \nu_{3}\left(|x|_{\mathcal{W}_{x}}\right)+c_{3}
$$

Theorem 4.2 (Cascades of Strongly iISS systems). Let Assumptions 2 and 3 hold. If (H1) the driving system is Strongly iISS wrt input d and with input threshold $R_{x}$; (H2) the driven system is Strongly iISS wrt input $|X(\cdot)|$

\footnotetext{
${ }^{1}$ In the definition of $|x|$ for $x \in M_{x}$ we use the following result. For a Riemannian manifold $M_{x}$, the Euclidean metric is uniformly equivalent to the Riemannian metric, i.e. there exists $\nu_{1}, \nu_{2} \in \mathcal{K}_{\infty}$ such that $\nu_{1}\left(\mathfrak{d}\left[x, x_{0}\right]\right) \leq|x| \leq \nu_{2}\left(\mathfrak{d}\left[x, x_{0}\right]\right)$, with $x_{0}$ denoting the "origin" element of $M_{x}$. Therefore, we define $|x|$ as the Euclidean norm if $M_{x}$ is Euclidean, and $|x|:=\mathfrak{d}\left[x, x_{0}\right]$ if $M_{x}$ is not Euclidean, thus implicitly making use of $\nu_{1}, \nu_{2}$ in all subsequent proofs.
}

and with input threshold $R_{z}$; (H3) it holds that $R_{z}=c_{3}+\tilde{c}$ for some $\tilde{c}>0$; then (T1) $\mathcal{W}_{\Theta}$ qualifies as a $\mathcal{W}$-limit set for system (34) and admits a finite decomposition without cycles under the flow of (34); (T2) the cascade system is Strongly iISS wrt input $d$ and with input threshold $R:=$ $\min \left\{\kappa_{x}^{-1}\left(\nu_{3}^{-1}(\tilde{c})\right), R_{x}\right\}$, where $\kappa_{x} \in \mathcal{K}_{\infty}$ denotes the $A G$ gain of the driving system for small inputs.

Remark 6 Our previous example (Subsection 2.4.2) has remarked the importance of Assumption 1 and the filtration ordering to prove ISS of a given $\mathcal{W}$-limit set. It is then worth to observe that the filtration ordering of $\mathcal{W}_{\Theta}$ in both Theorems 4.1 and 4.2 is given by:

$$
\mathcal{W}_{z, h}^{(i)} \prec \mathcal{W}_{z, k}^{(i)} \Leftrightarrow\left\{x_{i}\right\} \times \mathcal{W}_{z, h}^{(i)} \prec\left\{x_{i}\right\} \times \mathcal{W}_{z, k}^{(i)},
$$

for all $i \in\{1, \ldots, K\}$, all $h \in\left\{1, \ldots, K_{i}\right\}$, and all $k \in$ $\left\{1, \ldots, K_{i}\right\}$.

\subsection{Multiperiodicity}

In this Subsection, we move our attention to a wider class of attractors to which the driving subsystem (33a) converges, namely periodic orbits.

A periodic orbit $\Gamma_{x, j}, j=1, \ldots, N_{p}$ of the driving system (34a) will denote a compact invariant subset satisfying the following property:

$$
\begin{aligned}
& \forall i=1, \ldots, N_{p} \exists T_{i}>0 \quad \text { such that } \\
& X\left(t+T_{i}, x ; 0\right)=X(t, x ; 0) \forall x \in \Gamma_{i} \forall t \in \mathbb{R} .
\end{aligned}
$$

For the analysis of this scenario, a very natural option is to consider the notion of incremental ISS [4], which we briefly recall here.

Definition 4.3. (Incremental ISS) System (34b) is said to be incrementally input-to-state stable $(\delta I S S)$ if the state manifold is the Euclidean space $\left(M_{z}=\mathbb{E}^{n_{z}}\right)$ and there exists a $\mathcal{K} \mathcal{L}$ function $\beta$ and $\gamma \in \mathcal{K}_{\infty}$ such that for any $t \geq 0$, any $z_{1}, z_{2} \in \mathbb{R}^{n_{z}}$ and any couple of input signals $X_{1}(\cdot), X_{2}(\cdot)$, the following estimate holds true:

$$
\begin{aligned}
& \left|Z\left(t, z_{1} ; X_{1}\right)-Z\left(t, z_{2} ; X_{2}\right)\right| \leq \\
& \quad \beta\left(\left|z_{1}-z_{2}\right|, t\right)+\gamma\left(\mathfrak{d}_{[0,+\infty)}\left[X_{1}(\cdot), X_{2}(\cdot)\right]\right) .
\end{aligned}
$$

Without loss of generality, is is assumed $f(0,0)=0$.

Incremental ISS belongs to the palette of approaches (such as contraction analysis [35], convergent dynamics [39], or incremental stability in the framework of InputOutput operators theory [47]) to systems which exhibit incremental stability properties in presence of inputs. The adjective "incremental" here refers to the fact that solutions of a dynamical systems are compared with one another, rather than with a single special trajectory of interest (such as an equilibrium point). It turns out the incremental stability properties are especially suited to achieve synchronization of dynamical systems [56] and entrainment to periodic inputs $[4,46]$.

We are now going to introduce sufficient conditions for the ISS stability of the cascade system (33), based on the following two key assumptions. 
Assumption 4. (Multiperiodicity without cycles of driving system) The driving system (34a) satisfies the no-cycle condition and, moreover, the decomposition of its $\mathcal{W}$-limit set $\mathcal{W}_{x}$ reads as the disjoint union of the following sets:

$$
\bigcup_{k=1}^{N_{p}+N_{f}} \mathcal{W}_{x, k}=\bigcup_{j=1}^{N_{p}} \Gamma_{x, j}+\bigcup_{h=1}^{N_{f}}\left\{x_{h}\right\}
$$

where $\Gamma_{x, j} \subset M_{x}$ for $j=1, \ldots, N_{p}$ is a periodic orbit, and $x_{i} \in M_{x}$ for $h=1, \ldots, N_{f}$ is an isolated fixed point.

Assumption 5. (Incremental ISS of driving system) System (34b) is incrementally ISS.

In this framework, it makes sense to study the response of an incremental ISS system under the different types of forcing signals in consideration. As mentioned before, incremental ISS entails entrainment to constant inputs and periodic orbits, as detailed in the following:

- if the input converges to a constant signal, then the state converges to a unique equilibrium point. In case of system (33b), we will have that if $X(t, x ; 0) \rightarrow$ $x_{i}$ as $t \rightarrow+\infty$ for some $x_{i}$ as in (39), then there exists a unique equilibrium point $z_{i} \in M_{z}$ such that $Z(t, z ; X(\cdot)) \rightarrow z_{i}$ as $t \rightarrow+\infty$

- if the input converges to a periodic signal, then the state converges to a unique periodic orbit with same phase; in particular, shifting the phase of the periodic input signal will shift the phase of the corresponding state periodic orbit. In case of system (33b), we will consider the periodic input $X^{(i)}(t)$ as in (39), and the initial condition $z^{(i), 0}$ such that $Z^{(i)}(t):=Z\left(t, z^{(i), 0}, X^{(i)}(\cdot)\right)$ is the periodic state response of subsystem $(34 \mathrm{~b})$ to input $X^{(i)}(t)$. Existence of such initial condition is proved in [4, Proposition 4.4]. Now, if for some $x \in M_{x}$ and some $\theta \in[0,2 \pi)$ it holds that

$$
\lim _{t \rightarrow+\infty} \mathfrak{d}\left[X(t, x ; 0), X^{(i)}\left(t+\frac{\theta}{2 \pi} T_{i}\right)\right]=0,
$$

then

$$
\lim _{t \rightarrow+\infty}\left|Z(t, z ; X(\cdot, x ; 0))-Z^{(i)}\left(t+\frac{\theta}{2 \pi} T_{i}\right)\right|=0
$$

for all $z \in M_{z}$.

As done in Section 2, our goal is to find a $\mathcal{W}$-limit set for the cascade system (34) whose decomposition is the finest possible and, at the same time, satisfies the no-cycle condition under the flow of (34). To the aim of characterizing such set, we consider:

$$
\mathcal{W}_{\Theta}:=\bigcup_{j=1}^{N_{p}} \Gamma_{j} \cup \bigcup_{h=1}^{N_{f}}\left\{y_{h}\right\}
$$

where $\Gamma_{i}:=\left\{\left(\begin{array}{l}X^{(i)}(t) \\ Z^{(i)}(t)\end{array}\right), t \in\left[0, T_{i}\right)\right\}$ is the periodic orbit corresponding to $\Gamma_{x, i}$ in the decomposition (39) for all $i=$ $1, \ldots, N_{p}$, whereas $y_{i}:=\left(x_{i}, z_{i}\right)$ is the isolated fixed point corresponding to $\left\{x_{i}\right\}$ in the decomposition (39) for all $i=1, \ldots, N_{f}$.

We are now ready to state the main result of this Section.

Theorem 4.4 (Cascades of ISS systems - multiperiodic case [23]). Let Assumptions 4 and 5 hold. If the driving system (33a) is ISS wrt input d and the set $\mathcal{W}_{x}$, then (T1) the set $\mathcal{W}_{\Theta}$ qualifies itself as a $\mathcal{W}$-limit set and admits a finite decomposition without cycles (in the sense of Assumption 1) under the flow of (34); (T2) the cascade system (33) is ISS wrt input d and the set $\mathcal{W}_{\Theta}$.

Remark 7 The filtration ordering is inherited here as:

$$
\mathcal{W}_{x, i} \prec \mathcal{W}_{x, j} \Leftrightarrow \mathcal{W}_{i} \prec \mathcal{W}_{j}
$$

for all $i, j \in\{1, \ldots, K\}$.

\subsubsection{Example - The nonlinear pendulum}

Consider the nonlinear pendulum example in (32) with $b(\omega):=b \omega^{2} /\left(1+\omega^{2}\right)$.

\subsection{Example - Central Pattern Generators in robotics}

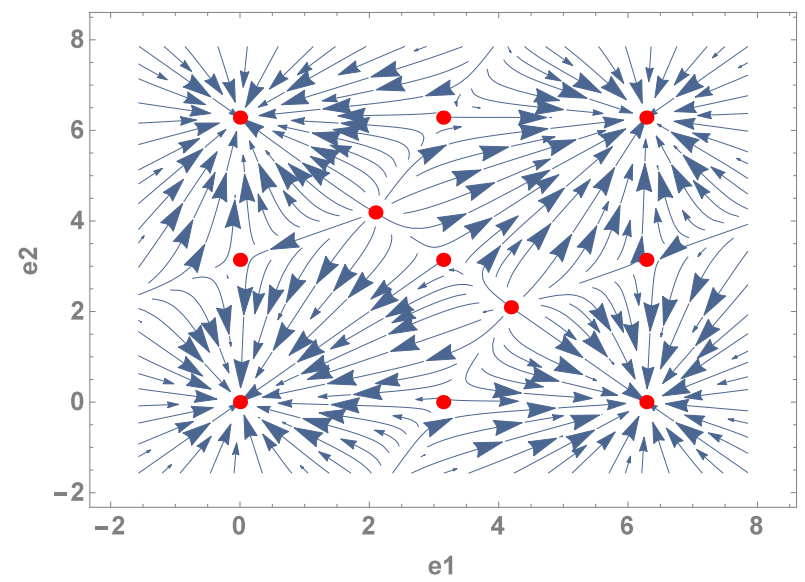

Figure 3: Phase plot of dynamics (46) in the coordinates $e_{1}, e_{2}$. The set $\mathcal{W}_{e}$ as in (47) is depicted in red.

As an example of an incrementally ISS system driven by an oscillating source, we study here the case of a robotic manipulator tracking a trajectory provided by the so-called Central Pattern Generators (CPGs). CPGs represent a powerful method for the robust generation of rhythmic patterns and for this reason they are widely used in locomotion control of robots [27]. Consider a 3 degreesof-freedom mechanical manipulator whose motion is described by the equations:

$$
M(q) \ddot{q}+C(q, \dot{q}) \dot{q}+G(q)=\tau,
$$


with $q, \dot{q}, \ddot{q} \in \mathbb{R}^{3}$ the joint angles, velocities and accelerations, $M(q)$ the inertia matrix, $C(q, \dot{q})$ the Coriolis matrix, $G(q)$ the potential vector field, $\tau$ the vector of all available control torques, and $d$ disturbances occurring in the joint dynamics. The standard computed torque control algorithm is implemented on (41):

$$
\begin{aligned}
& \tau=M(q) \nu+C(q, \dot{q}) \dot{q}+G(q) \\
& \nu=q_{r(2)}+K_{d}\left(q_{r(1)}-\dot{q}\right)+K_{p}\left(q_{r}-q\right),
\end{aligned}
$$

where $K_{d}=\operatorname{diag}\left[k_{d 1}, k_{d 2}, k_{d 3}\right]$ and $K_{p}=\operatorname{diag}\left[k_{p 1}, k_{p 2}, k_{p 3}\right]$ are tunable controller gains. In (42), variables

$$
q_{r}=\left(\begin{array}{l}
q_{r 1} \\
q_{r 2} \\
q_{r 3}
\end{array}\right), q_{r(1)}=\left(\begin{array}{l}
q_{r 1(1)} \\
q_{r 2(1)} \\
q_{r 3(1)}
\end{array}\right), q_{r(2)}=\left(\begin{array}{l}
q_{r 1(2)} \\
q_{r 2(2)} \\
q_{r 3(1)}
\end{array}\right)
$$

respectively denote the reference trajectory and its filtered first and second time derivative, as specified in the following:

$$
\frac{q_{r i(1)}(s)}{q_{r i}(s)}=\frac{p s}{p+s}, \frac{q_{r i(2)}(s)}{q_{r i}(s)}=\frac{p s^{2}}{(p+s)^{2}},
$$

with $i=1,2,3$ and $p>0$ the tunable pole of the filter. By combining equations (41), (42), and a minimal statespace representation of (44), we obtain the following linear system for each joint $i=1,2,3$ :

$$
\dot{z}=A z+B q_{r}
$$

with

$$
A=\left[\begin{array}{cccc}
-p & 0 & 0 & 0 \\
-p^{2} & -p & 0 & 0 \\
0 & 0 & 0 & 1 \\
k_{d i}+p & 1 & -k_{p i} & -k_{d i}
\end{array}\right], B=\left[\begin{array}{c}
-p^{2} \\
-p^{3} \\
0 \\
p^{2}+k_{p i}+k_{d i} p
\end{array}\right]
$$

The reference trajectory $q_{r}$ for the torque-controlled manipulator is generated by the CPGs, which are implemented as a set of coupled nonlinear oscillators (typically $\mathrm{Ku}-$ ramoto model), as follows:

$$
\begin{aligned}
\dot{\theta_{1}} & =\omega+d_{1}+K \sin \left(\theta_{2}-\theta_{1}\right)+K \sin \left(\theta_{3}-\theta_{1}+\pi\right) \\
\dot{\theta_{2}} & =\omega+d_{2}+K \sin \left(\theta_{1}-\theta_{2}\right)+K \sin \left(\theta_{3}-\theta_{2}+\pi\right) \\
\dot{\theta_{3}} & =\omega+d_{3}+K \sin \left(\theta_{1}-\theta_{3}-\pi\right)+K \sin \left(\theta_{2}-\theta_{3}-\pi\right) \\
q_{r i} & =\bar{q}_{i}+\hat{q}_{i} \cos \left(\theta_{i}\right)
\end{aligned}
$$

where $\theta_{i} \in \mathbb{S}$ denotes the phase of oscillator $i=1,2,3$, $\omega>0$ is the common frequency for all oscillators, $d_{i} \in \mathbb{R}$ represents a generic disturbance, $K>0$ is the coupling strength, and $\bar{q}_{i}, \hat{q}_{i} \in \mathbb{R}$ respectively denote bias and amplitude of the oscillation driving joint $i=1,2,3$. Model (46) has been studied in [7] where the authors have shown that, by rewriting the dynamics wrt the synchronization errors $e_{1}:=\theta_{2}-\theta_{1}, e_{2}:=\theta_{3}-\theta_{1}+\pi$, the $\mathcal{W}$-limit set for the transformed dynamics takes the form:

$$
\mathcal{W}_{e}=\left\{(0,0),(0, \pi),(\pi, 0),(\pi, \pi),\left(\frac{2 \pi}{3}, \frac{4 \pi}{3}\right),\left(\frac{4 \pi}{3}, \frac{2 \pi}{3}\right)\right\}
$$

and, moreover, satisfies the no cycle condition (1), as shown in Figure 3. This in turn implies that the $\mathcal{W}$-limit set $\mathcal{W}_{x}$ of the original system (46) consists of periodic orbits in a one-to-one correspondence with the elements of (47) and satisfies the no cycle condition.

The authors in [7] have proved that system (46) is ISS wrt to disturbances $d_{i}$ and the set $\mathcal{W}_{x}$. It can be easily proved that system $\dot{z}=A z+B\left(\bar{q}_{i}+\hat{q}_{i} \cos \left(\theta_{i}\right)\right)$ is incrementally ISS wrt to input $\theta_{i}$ by noticing that it is a linear system and $\left|\cos \left(\theta_{i}\right)\right| \leq 1$ is bounded. Therefore the results of Theorem 4.4 can be applied to infer the ISS property for the cascade (45), (46) wrt disturbances $d_{i}$.

\section{Conclusions and open research directions}

This paper has surveyed a number of recent results on the ISS approach to the analysis of stability and robustness of nonautonomous systems with decomposable invariant sets and evolving on manifolds. We have shown how the classical notions of ISS, integral ISS, and Strong iISS can be generalized in the context of multistable systems, whenever a decomposition for its compact invariant set is selected so as to exhibit no cycle among the components of the decomposition itself. Further results on the conservation of the ISS property under cascade interconnection have been provided.

A number of open research directions can be envisioned within this novel ISS framework for multistable systems. It is worth to mention two of them, as follows.

Feedback of ISS systems with multiple invariant sets). The two fundamental interconnections for the stability analysis of large networks are cascades and feedback systems. In the context of classical ISS theory, both types of interconnections are well understood. In regard to feedback interconnections in particular, a basic contribution has been established with a small gain theorem [33] and its related Lyapunov formulation [32]. Conversely in the context of ISS for multistable systems, while cascades display properties that are somehow similar to the ones displayed by classical ISS systems, the study of feedback interconnections seems a far more challenging area to research. Indeed, consider the following setting:

$$
\begin{aligned}
& \dot{x}_{1}(t)=f_{1}\left(x_{1}(t), x_{2}(t)\right) \\
& \dot{x}_{2}(t)=f_{2}\left(x_{2}(t), x_{1}(t)\right)
\end{aligned}
$$

where $f_{1}\left(x_{1}, x_{2}\right): M_{1} \times M_{2} \rightarrow T_{x_{1}} M_{1}, f_{2}\left(x_{2}, x_{1}\right): M_{2} \times$ $M_{1} \rightarrow T_{x_{2}} M_{2}$ are two class $\mathcal{C}^{1}$ mappings, and $M_{1}, M_{2}$ respectively are two connected Riemannian manifolds without boundary. We denote by $\mathcal{W}_{1}$ and $\mathcal{W}_{2}$ the compact invariant sets containing all $\alpha$ - and $\omega$-limit sets of systems $\dot{x}_{1}=f_{1}\left(x_{1}, 0\right)$ and $\dot{x}_{2}=f_{2}\left(x_{2}, 0\right)$ respectively. In this setting, a novel and remarkably non-trivial obstacle is represented by the definition itself of the $\mathcal{W}$-limit set of the feedback system (48). However, assuming ISS for each subsystem (48a) and (48b) yields boundedness of trajectories of the feedback interconnection (48) under suitable smallgain-like conditions, though we do not prove this result 
here. This research direction has been partially addressed by the contributions $[3,2]$.

ISS-related stability notions) The classical ISS framework encompasses a number of different stability notions [51] such as: ISS with respect to Input Derivatives, InputOutput Stability, Input-Measurement-to-Error Stability, Output-to-Input Stability. Extending each of these notions to multistable systems would contribute in expanding the current novel framework.

\section{Acknowledgment}

The authors would like to thank the anonymous reviewers for their valuable comments and suggestions to improve the quality of the paper.

[1] Abraham, R., Marsden, J., Ratiu, T., 1988. Manifolds, Tensor Analysis, and Applications. Springer-Verlag, New York.

[2] Ahmed, H., Ushirobira, R., Efimov, D., Perruquetti, W., July 2015. On conditions of robust synchronization for multistable systems. In: Control Conference (ECC), 2015 European. pp. $181-185$.

[3] Ahmed, H., Ushirobira, R., Efimov, D., Perruquetti, W., 2015. Robust synchronization for multistable systems. IEEE Transactions on Automatic Control PP (99), 1-1.

[4] Angeli, D., Mar 2002. A Lyapunov approach to incremental stability properties. Automatic Control, IEEE Transactions on 47 (3), 410-421.

[5] Angeli, D., Dec 2003. Some remarks on density functions for dual Lyapunov methods. In: Decision and Control, 2003. Proceedings. 42nd IEEE Conference on. Vol. 5. pp. 5080-5082 Vol.5.

[6] Angeli, D., June 2004. An almost global notion of Input-to-State Stability. Automatic Control, IEEE Transactions on 49 (6), 866-874.

[7] Angeli, D., Efimov, D., Dec 2015. Characterizations of Input-toState Stability for Systems With Multiple Invariant Sets. IEEE Transactions on Automatic Control 60 (12), 3242-3256.

[8] Angeli, D., Ingalls, B., Sontag, E., Wang, Y., 2004. Separation Principles for Input-Output and Integral-Input to State Stability. SIAM J. Control Optim 43, 256-276.

[9] Angeli, D., Praly, L., July 2011. Stability robustness in the presence of exponentially unstable isolated equilibria. IEEE Transactions on Automatic Control 56 (7), 1582-1592.

[10] Angeli, D., Sontag, E., Wang, Y., Jun 2000. A characterization of Integral Input-to-State Stability. Automatic Control, IEEE Transactions on 45 (6), 1082-1097.

[11] Angeli, D., Sontag, E. D., Wang, Y., April 2000. Further Equivalences and Semiglobal Versions of Integral Input to State Stability. Dyn. Control 10 (2), 127-149.

[12] Arcak, M., Angeli, D., Sontag, E., 2002. A Unifying Integral ISS Framework For Stability Of Nonlinear Cascades. SIAM J. Control Optim 40, 1888-1904.

[13] Boothby, W. M., 1975. An Introduction to Differentiable Manifolds and Riemannian Geometry. Academic Press, New York.

[14] Chaillet, A., Angeli, D., Ito, H., Sept 2014. Combining iISS and ISS With Respect to Small Inputs: The Strong iISS Property. Automatic Control, IEEE Transactions on 59 (9), 2518-2524.

[15] Chaillet, A., Angeli, D., Ito, H., 2014. Strong iISS is preserved under cascade interconnection. Automatica 50 (9), $2424-2427$.

[16] do Carmo, M. P., 1992. Riemannian Geometry. Birkhuser, Birkhuser Basel.

[17] Efimov, D., 2012. Global Lyapunov Analysis of Multistable Nonlinear Systems. SIAM Journal on Control and Optimization 50 (5), 3132-3154.

[18] Efimov, D., Ortega, R., Schiffer, J., July 2015. ISS of multistable systems with delays: Application to droop-controlled inverterbased microgrids. In: American Control Conference (ACC), 2015. pp. 4664-4669.
[19] Efimov, D., Schiffer, J., Ortega, R., 2016. Robustness of delayed multistable systems with application to droop-controlled inverter-based microgrids. International Journal of Control 89 (5), 909-918.

[20] Enciso, G., Sontag, E. D., 2005. Monotone systems under positive feedback: multistability and a reduction theorem. Systems \& Control Letters 54 (2), $159-168$.

[21] Forni, P., Angeli, D., Dec 2016. Cascades of iISS and Strong iISS systems with multiple invariant sets. In: 2016 55th IEEE Conference on Decision and Control (CDC). pp. 3742-3747.

[22] Forni, P., Angeli, D., 2016. Characterizations of Integral Inputto-State Stability for systems with multiple invariant sets. Automatic Control, IEEE Transactions on.

[23] Forni, P., Angeli, D., 2016. Input-to-State Stability for cascade systems with multiple invariant sets. Systems \& Control Letters.

[24] Forni, P., Angeli, D., 2016. Output-to-State Stability for systems with decomposable invariant sets. In: Decision and Control, 2016. 55th IEEE Conference on.

[25] Gelig, A. K., Leonov, G. A., Iakubovich, V. A., 1978. Stability of nonlinear systems with nonunique equilibrium position. Moscow Izdatel Nauka.

[26] Guerra, M., Zheng, G., Efimov, D., Perruquetti, W., July 2015. An ISS based solution to avoid local minima in the Potential Field method. In: Control Conference (ECC), 2015 European. pp. 1974-1979.

[27] Ijspeert, A. J., 2008. Central pattern generators for locomotion control in animals and robots: A review. Neural Networks $21(4), 642-653$, robotics and Neuroscience.

[28] Ito, H., 2001. New characterization and solution of input-tostate stabilization: a state-dependent scaling approach. Automatica 37 (10), 1663 - 1670.

[29] Izhikevich, E. M., 2006. Dynamical Systems in Neuroscience: The Geometry of Excitability and Bursting. The MIT Press, Cambridge, Massachusetts.

[30] Jiang, Z.-P., Khorrami, F., Hill, D., 1999. Decentralized outputfeedback control with disturbance attenuation for large-scale nonlinear systems. In: Decision and Control, 1999. Proceedings of the 38th IEEE Conference on. Vol. 4. pp. 3271-3276 vol.4.

[31] Jiang, Z.-P., Mareels, I., Hill, D., Huang, J., April 2004. A unifying framework for global regulation via nonlinear output feedback: from ISS to iISS. Automatic Control, IEEE Transactions on 49 (4), 549-562.

[32] Jiang, Z.-P., Mareels, I. M. Y., Wang, Y., August 1996. A Lyapunov Formulation of the Nonlinear Small-gain Theorem for Interconnected ISS Systems. Automatica 32 (9), 1211-1215.

[33] Jiang, Z.-P., Teel, A. R., Praly, L., 1994. Small-gain theorem for ISS systems and applications. Mathematics of Control, Signals and Systems 7 (2), 95-120.

[34] Liberzon, D., 1999. ISS and integral-ISS disturbance attenuation with bounded controls. In: Decision and Control, 1999. Proceedings of the 38th IEEE Conference on. Vol. 3. pp. 25012506 vol.3.

[35] Lohmiller, W., Slotine, J.-J. E., 1998. On contraction analysis for non-linear systems. Automatica 34 (6), 683 - 696.

[36] Mancilla-Aguilar, J., García, R., 2001. On converse Lyapunov theorems for ISS and iISS switched nonlinear systems. Systems \& Control Letters $42(1), 47-53$.

[37] Moser, J., July 1961. Bistable systems of differential equations with applications to tunnel diode circuits. IBM Journal of Research and Development 5 (3), 226-240.

[38] Nitecki, Z., Shub, M., 1975. Filtrations, decompositions, and explosions. American Journal of Mathematics 97 (4), pp. 10291047.

[39] Pavlov, A., Pogromsky, A., van de Wouw, N., Nijmeijer, H., 2004. Convergent dynamics, a tribute to boris pavlovich demidovich. Systems \& Control Letters 52 (34), 257 - 261.

[40] Pisarchik, A. N., Feudel, U., 2014. Control of multistability. Physics Reports 540 (4), 167 - 218, control of multistability.

[41] Prajna, S., Parrilo, P., Rantzer, A., Feb 2004. Nonlinear control synthesis by convex optimization. Automatic Control, IEEE Transactions on 49 (2), 310-314. 
[42] Praly, L., Jiang, Z.-P., Jun. 1993. Stabilization by Output Feedback for Systems with ISS Inverse Dynamics. Syst. Control Lett. 21 (1), 19-33.

[43] Pugh, C., Shub, M., 1970. Omega stability theorem for flows. Inventiones Math. 158, pp. 11-150.

[44] Rantzer, A., 2001. A dual to Lyapunov's stability theorem. Systems \& Control Letters 42 (3), 161-168.

[45] Rantzer, A., Dec 2002. An converse theorem for density functions. In: Decision and Control, 2002, Proceedings of the 41st IEEE Conference on. Vol. 2. pp. 1890-1891 vol.2.

[46] Russo, G., di Bernardo, M., Sontag, E. D., 04 2010. Global entrainment of transcriptional systems to periodic inputs. PLoS Comput Biol 6 (4), 1-26.

[47] Sandberg, I. W., Jul 1992. Approximately-finite memory and input-output maps. IEEE Transactions on Circuits and Systems I: Fundamental Theory and Applications 39 (7), 549-556.

[48] Sontag, E., Wang, Y., 2000. Lyapunov Characterizations of Input to Output Stability. SIAM Journal on Control and Optimization 39 (1), 226-249.

[49] Sontag, E. D., Apr 1989. Smooth stabilization implies coprime factorization. Automatic Control, IEEE Transactions on 34 (4), 435-443.

[50] Sontag, E. D., 1998. Comments on integral variants of ISS. Systems \& Control Letters 34 (12), $93-100$.

[51] Sontag, E. D., 2006. Input to state stability: Basic concepts and results. In: Nonlinear and Optimal Control Theory. Springer, pp. $163-220$.

[52] Sontag, E. D., Teel, A., Aug 1995. Changing supply functions in input/state stable systems. Automatic Control, IEEE Transactions on 40 (8), 1476-1478.

[53] Sontag, E. D., Wang, Y., 1995. On characterizations of the input-to-state stability property. Systems \& Control Letters 24 (5), $351-359$.

[54] Sontag, E. D., Wang, Y., Sep 1996. New characterizations of input-to-state stability. Automatic Control, IEEE Transactions on 41 (9), 1283-1294.

[55] Sontag, E. D., Wang, Y., 1999. Notions of input to output stability. Systems \& Control Letters 38 (45), 235 - 248.

[56] Wang, W., Slotine, J.-J. E., Jan. 2005. On partial contraction analysis for coupled nonlinear oscillators. Biol. Cybern. 92 (1), $38-53$. 